\title{
Follicular Aspiration Is Superior to Coasting as Effective Prophylactic Procedure against Ovarian Hyperstimulation Syndrome
}

\author{
Walid Mohamed Elnagar1 ${ }^{*}$, Huda Fathy Ebian² \\ ${ }^{1}$ Obstetrics and Gynecology Department, Faculty of Medicine, Zagazig University, Zagazig, Egypt \\ ${ }^{2}$ Clinical Pathology Department, Faculty of Medicine, Zagazig University, Zagazig, Egypt \\ Email: *whitewhale1977@gmail.com
}

How to cite this paper: Elnagar, W.M. and Ebian, H.F. (2019) Follicular Aspiration Is Superior to Coasting as Effective Prophylactic Procedure against Ovarian Hyperstimulation Syndrome. Open Journal of Obstetrics and Gynecology, 9, 679-690.

https://doi.org/10.4236/ojog.2019.95067

Received: April 23, 2019

Accepted: May 19, 2019

Published: May 22, 2019

Copyright (๑) 2019 by author(s) and Scientific Research Publishing Inc. This work is licensed under the Creative Commons Attribution International License (CC BY 4.0).

http://creativecommons.org/licenses/by/4.0/

c) (i) Open Access

\begin{abstract}
Objectives: Evaluation of outcome follicular aspiration compared to coating procedure for infertile women undergoing ICSI regarding frequency and severity of ovarian hyperstimulation syndrome (OHSS). Patients \& Methods: Infertile PCOS women underwent controlled ovarian stimulation with luteal phase GnRH-agonist long down-regulation protocol. Women developed serum $E_{2}$ level $>4000 \mathrm{pg} / \mathrm{ml}$ and/or $>20$ follicles of $\geq 10 \mathrm{~mm}$ in diameter were randomly divided to receive coasting strategy (Coasting group) or TVU-guided aspiration (Aspiration group). When $\geq 3$ follicles were $\geq 18 \mathrm{~mm}$ and serum $\mathrm{E}_{2}$ level was $<3000 \mathrm{pg} / \mathrm{ml}, \mathrm{GnRH}$ agonist was continued, and $10,000 \mathrm{IU}$ was administered $36 \mathrm{~h}$ before oocyte retrieval, and ICSI was performed 72-hr thereafter and the frequency of moderate-to-severe OHSS was determined. Results: 82 women developed criteria for categorization and 21 women (25.6\%) developed moderate-to-severe OHSS; 5 women of aspiration and 16 of Coasting group; with significantly $(\mathrm{P}=0.005)$ lower frequency in aspiration group. Twenty women developed ascites; 3 women had clinically detectable, while 17 women had US detected ascites with significantly $(\mathrm{P}=$ 0.039) higher frequency among women that had coasting. All other manifestations of OHSS were significantly lower with aspiration procedure. Both coasting and aspiration therapy significantly reduced serum E2 and ovarian diameter on day of hCG injection compared to estimates taken at time of categorization. Conclusion: Coasting procedure prior to hCG injection could decrease incidence of OHSS and lessens its manifestation. Follicular aspiration provided more superior results and improved outcome of these women. Any of these modalities could be provided to infertile high-risk women according to the availability of experiences and patients' selection.
\end{abstract}




\section{Keywords}

Ovarian Hyperstimulation Syndrome, PCOS, Follicular Aspiration, Coasting Procedure

\section{Introduction}

Ovarian stimulation (OS) is crucial for pregnancy success in assisted reproductive technology treatments [1]. However, ovarian hyperstimulation syndrome (OHSS) in hyper-responders to OS is common despite being self-limited [2]. It is a severe complication for patients undergoing IVF/ICSI [3]. Vascular endothelial growth factor (VEGF) plays the most important role in pathogenesis of OHSS and is related to concomitant increased capillary permeability and fluid retention [2].

Presence of genetic mutations is already known to be involved in OHSS [4]. Women who had follicle-stimulating hormone receptor variant asparagine/serine in amino acid 680 are at higher risk for OHSS during IVF [5]. Also, a potential link was suggested between OHSS and mutations of the FLT4 gene which encodes Fms-related tyrosine kinase 4 [4]. Production of some pro-inflammatory molecules such as IL-8, IL-1, IL-6 and TNF- $\alpha$ was incriminated in pathogenesis of OHSS [2].

Therapeutic options to reduce the incidence and lessen the severity of OHSS are variant and with contradictory results [6]. The "freeze-all" strategy provides benefit for high-responder women who were at risk of OHSS, but should not be offered universally [7]. Addition of hp-hMG on day of antagonist initiation significantly lowers OHSS rates [8]. In sequential ovarian stimulation cycles, individualized doses of follitropin delta decreased the risk of moderate/severe OHSS than conventional follitropin alfa regimen [9].

Multiple drugs were evaluated for prophylaxis against or treatment of OHSS [10]. In rat model of OHSS, administration of myo-inositol and metformin significantly reduced VEGF expression and decreased vascular permeability [11]. Prophylactic vitamin D supplemental therapy effectively increased pigment epithelium-derived factor which protected against OHSS [12]. Montelukast [13] and resveratrol [14] induced reduction of ovarian diameter and VEGF expression.

Coasting is a well-known strategy to decrease severity of OHSS [15] and acceleration of coasting in cases of OHSS through treatment with GnRH-ant after pituitary suppression with GnRH reduces estradiol (E2) level, and avoids cycle cancellation with prevention of OHSS [16]. Thus, the current study tried evaluation of outcome follicular aspiration compared to coating procedure for infertile women undergoing ICSI regarding the frequency and severity of OHSS.

\section{Design}

Prospective comparative clinical trial. 


\section{Setting}

Department of Obstetrics \& Gynecology, and clinical pathology, Faculty of Medicine, Zagazig University.

\section{Patients \& Methods}

The study protocol was approved by the Local Ethical Committee. All PCOS infertile women assigned to undergo ICSI cycles since June 2016 were eligible for evaluation. Inclusion criteria included the presence of at least 20 pre-ovulatory follicles in both ovaries with serum E2 level $\geq 3000 \mathrm{pg} / \mathrm{ml}$ in PCOS women. PCOS was diagnosed according to Rotterdam criteria depending on the presence of at least two of oligomenorrhic and/or anovulatory cycles, clinical and/or biochemical signs of hyperandrogenemia with serum total testosterone level $>0.8$ $\mathrm{ng} / \mathrm{ml}$ and polycystic ovaries containing $>12$ follicles of $2-9 \mathrm{~mm}$ in diameter and/or an ovarian volume $>10 \mathrm{ml}$ per ovary by vaginal ultrasound [17]. Exclusion criteria included BMI $>30 \mathrm{~kg} / \mathrm{m}^{2}$, history of previous development of OHSS, endocrinopathies, maintenance on insulin-sensitizing drugs, receiving GnRH antagonists.

All women underwent controlled ovarian stimulation (COS) with luteal phase GnRH-agonist long down-regulation protocol using subcutaneous injection of Triptorelin acetate 0.1 daily (DecapeptylGynTM; Ferring, Kiel, Germany) on $21^{\text {st }}$ day of cycle that precede stimulation. Adequate pituitary desensitization was established on $2^{\text {nd }}$ or $3^{\text {rd }}$ day of menses and ovarian stimulation with gonadotropins was started on $3^{\text {rd }}$ day of the oncoming cycle by the use of recombinant-FSH (r-hFSH, Gonal-F®, 75 UI, Serono, Switzerland) at a daily dose 75 $150 \mathrm{IU}$ according to the individual response to treatment for 5 - 7 days. Follow-up consisted of estimation of serum E2 every 2 days and ultrasonographicscanning by $7-10 \mathrm{MHZ}$ probe (Voluson $730 \mathrm{PRO} \mathrm{V}$, GE Healthcare, USA) was done every 3 - 5 days.

Women developed serum $\mathrm{E}_{2}$ level of $>4000 \mathrm{pg} / \mathrm{ml}$ and/or sonographic detection of $>20$ follicles each of $\geq 10 \mathrm{~mm}$ in diameter and at least $20 \%$ of follicles were $\geq 15 \mathrm{~mm}$ diameter were included in the trial and were randomly, using sealed envelops, divided into two equal groups: Coasting group included women underwent coasting strategy and Aspiration group included women underwent follicular aspiration. For coasting group, serum $\mathrm{E}_{2}$ and FSH levels were measured from the day of coasting until the day of hCG administration. For women of aspiration group, TVU-guided oocyte retrieval was performed under propofol sedation using single-lumen 17-gauge needle (Cook Ireland, Limerick, Ireland) at maintained pressure of $80 \mathrm{mmHg}$.

When at least three follicles reached a mean diameter of $\geq 18 \mathrm{~mm}$ and serum $\mathrm{E}_{2}$ level was $<3000 \mathrm{pg} / \mathrm{ml}$, GnRH agonist was continued and a single intramuscular injection of 10,000 IU hCG (Choriomon, IBSA, Switzerland) was administered $36 \mathrm{~h}$ before the planned time of oocyte retrieval and then ICSI was performed, 72-hr thereafter, a maximum of two embryos were transferred, as described previously by Huisman et al. [18]. All embryos were scored according to 
Steer et al. [19] on day of transfer and embryos in G1 and G2 grade having 4 blastomeres on day 2 or $\geq 8$ blastomeres on day 3, less than $20 \%$ fragmentation, and no multinuclear blastomeres were transferred. Luteal phase support was started the day after ovum pick up by the vaginal administration of progesterone (Cyclogest $200 \mathrm{mg}$ suppositories; Actavis UK Limited) twice daily for 16 days and was continued for up to 12 weeks if pregnancy occurred.

\section{Diagnosis and Classification of OHSS}

OHSS was diagnosed according to the criteria of Golan et al. [20] as mild OHSS on presence of abdominal distension and discomfort (Grade 1) with nausea, vomiting and/or diarrhea and ovarian enlargement up to $12 \mathrm{~cm}$ (Grade 2). Moderate OHSS (Grade 3) was diagnosed in patients had clinical presentation of grade 2 OHSS plus ultrasonographic evidence of ascites. OHSS was considered severe (Grade 4) if there was clinical evidence of grade 3 OHSS plus clinical evidence of ascites and/or hydrothorax, breathing difficulties; and was considered as severe OHSS of grade 5 if patient of grade 4 developed increased blood viscosity due to hemoconcentration, as well asdecreasedrenal perfusion [21].

\section{Evaluated Parameters}

1) Primary outcome is the frequency of moderate-to-severe OHSS.

2) The secondary outcome included the following items:

- Presence and severity of abdominal pain was graduated using a numerical pain visual analogue scale (VAS) with 0 means no pain and 10 means severe intolerable pain [22].

- Nausea and/or vomiting and sense of abdominal distension were scored using verbal analogue scale as nil, mild, moderate, and severe symptom.

- Hospitalization rate and requirement of paracentesis.

\section{Statistical Analysis}

Obtained data were presented as mean $\pm \mathrm{SD}$, numbers and percentages. Results were analyzed using One-way ANOVA test, Student $\mathrm{t}$-test and Chi-square test $\left(\mathrm{X}^{2}\right.$ test). Statistical analysis was conducted using the IBM SPSS (Version 23, 2015) for Windows statistical package. $\mathrm{P}$ value $<0.05$ was considered statistically significant.

\section{Results}

Throughout the duration of the study, 82 women developed criteria for categorization (Figure 1). There were non-significant differences between women of both groups as regards basal data of enrolled women (Table 1).

At time of categorization, serum E2 levels increased significantly in all patients compared to their respective basal E2 levels. On day of hCG injection, estimated serum E2 levels significantly decreased in patients of both groups in comparison to levels estimated at time of categorization, but were still significantly higher compared to basal E2 levels. Serum E2 levels estimated at time of 
categorization were non-significantly lower in patients who underwent aspiration than patients undertook coasting procedure. On the other hand, serum E2 levels estimated on day of hCG injection were significantly lower in patients of aspiration group than in patients of coasting group (Figure 2). On day of hCG injection, serum FSH levels were significantly increased in all studied patients in comparison to corresponding levels estimated at time of categorization with non-significantly higher levels were estimated in women of aspiration group than women of coasting group (Table 2).

On day of hCG injection, ovarian diameter estimated in all studied patients was significantly decreased compared to their respective diameter determined at time of categorization with significantly smaller ovarian diameter in patients of aspiration group than in patients of coasting group (Table 2, Figure 3).

Considering the primary outcome; 21 women developed moderate-to-severe OHSS for a frequency of $25.6 \%$ (Figure 1). Seventeen women developed moderate OHSS and only four women developed severe OHSS of grade 4 and no women developed severe OHSS of grade 5. Only five women of aspiration group, while 16 women of Coasting group developed moderate-to-severe OHSS with significantly $(\mathrm{P}=0.005)$ lower frequency in aspiration group. The remaining 61 women developed mild OHSS; 36 and 25 in aspiration and coasting groups, respectively (Figure 4).

Twenty women developed ascites; 14 in coasting and 6 in aspiration groups. Three women; 2 in coasting and one in aspiration groups, had clinically detectableascites, while 17 women had US detected ascites clinically received coasting treatment with significantly $(P=0.039)$ lower frequency of ascites among women of aspiration group. All women developed abdominal distension of varied grades, but women received aspiration showed significantly $(P=0.002)$ lower distension score than women of coasting group. Nausea and vomiting occurred in 73 women and 47 women, respectively with significantly lower scores in women of aspiration versus coasting group. All women had pain with varied scores with significantly lower scores among women of aspiration group (Table 3). Twelve patients (14.6\%) required hospitalization; 8 patients in Coasting and 4 patients in aspiration group with non-significantly $(\mathrm{P}=0.211)$ lower frequency of hospitalization in aspiration group, but no patient required paracentesis.

Table 1. Data of studied women at time of evaluation.

\begin{tabular}{cccc}
\hline Group Data & Coasting $(\mathrm{n}=41)$ & Aspiration $(\mathrm{n}=41)$ & P value \\
\hline Age (years) & $27.5 \pm 4.3$ & $28.3 \pm 3.8$ & 0.522 \\
Weight $(\mathrm{kg})$ & $82 \pm 4.4$ & $80.8 \pm 5.6$ & 0.109 \\
Height $(\mathrm{cm})$ & $167.1 \pm 3.4$ & $166.3 \pm 4$ & 0.609 \\
BMI $\left(\mathrm{kg} / \mathrm{m}^{2}\right)$ & $29.4 \pm 1.3$ & $29.2 \pm 1.9$ & 0.327 \\
Duration of infertility (years) & $5.7 \pm 1.5$ & $6.2 \pm 2$ & 0.287 \\
Basal FSH level (IU/L) & $7.7 \pm 1.4$ & $7.5 \pm 1$ & 0.323 \\
Basal E E $_{2}$ level (pg/ml) & $3162.4 \pm 579$ & $3295.5 \pm 479$ & 0.219 \\
\hline
\end{tabular}

Data are presented as mean $\pm \mathrm{SD} ; \mathrm{P}>0.05$ indicates insignificant difference between both groups. 
Table 2. Data of studied women determined on day of hCG injection.

\begin{tabular}{|c|c|c|c|c|}
\hline \multicolumn{2}{|c|}{$\begin{array}{c}\text { Group } \\
\text { Variable Time }\end{array}$} & $\begin{array}{l}\text { Coasting } \\
(\mathrm{n}=41)\end{array}$ & $\begin{array}{c}\text { Aspiration } \\
(\mathrm{n}=41)\end{array}$ & $\mathrm{P}$ value \\
\hline \multirow{5}{*}{$\mathrm{E}_{2}$ level $(\mathrm{pg} / \mathrm{ml})$} & Basallevel & $3162.4 \pm 579$ & $3295.5 \pm 479$ & 0.219 \\
\hline & Categorization & $7150.5 \pm 1016$ & $6602 \pm 1495.4$ & 0.056 \\
\hline & Day of hCG injection & $6579.3 \pm 1240$ & $5827.2 \pm 1498.6$ & 0.015 \\
\hline & P1 & $<0.001$ & $<0.001$ & \\
\hline & $\mathrm{P} 2$ & 0.025 & 0.022 & \\
\hline \multirow{3}{*}{ FSH level (IU/L) } & Categorization & $7.7 \pm 1.4$ & $7.8 \pm 1.6$ & 0.719 \\
\hline & Day of hCG injection & $9 \pm 3.4$ & $9.7 \pm 3.8$ & 0.386 \\
\hline & $\mathrm{P} 2$ & 0.027 & 0.01 & \\
\hline \multirow{3}{*}{$\begin{array}{c}\text { Ovarian } \\
\text { diameter }(\mathrm{cm})\end{array}$} & Categorization & $14.9 \pm 5.8$ & $14.3 \pm 5.4$ & 0.461 \\
\hline & Day of hCG injection & $12.4 \pm 3.9$ & $10.5 \pm 4.7$ & 0.047 \\
\hline & P2 & 0.028 & 0.001 & \\
\hline
\end{tabular}

Data are presented as mean $\pm \mathrm{SD}$; P indicates insignificance of difference between both groups; P1: indicates significance of difference in E2 serum levels between basal and at categorization levels; P2: indicates significance of difference between measures estimated at time of categorization and on day of hCG injection; $\mathrm{P}<0.05$ : indicates significant difference; $\mathrm{P}>0.05$ : indicates non-significant difference.

Table 3. OHSS manifestations scoring of women enrolled in both groups.

\begin{tabular}{|c|c|c|c|c|c|c|c|}
\hline \multicolumn{3}{|c|}{ Manifestations } & \multicolumn{5}{|c|}{ Score } \\
\hline \multirow{4}{*}{$\begin{array}{l}\text { Abdominal } \\
\text { distension }\end{array}$} & \multirow{3}{*}{ Group } & items & 1 & 2 & 3 & 4 & Total \\
\hline & & Coasting & $7(17 \%)$ & $16(39 \%)$ & $17(41.5)$ & $1(2.5 \%)$ & $2.3 \pm 0.8$ \\
\hline & & Aspiration & $19(46.3 \%)$ & $14(34.2 \%)$ & $8(19.5 \%)$ & 0 & $1.7 \pm 0.7$ \\
\hline & \multicolumn{2}{|c|}{$P$ value } & \multicolumn{4}{|c|}{0.031} & 0.002 \\
\hline \multirow{4}{*}{ Nausea } & \multicolumn{2}{|c|}{ Score items } & 0 & 1 & 2 & 3 & Total \\
\hline & \multirow{2}{*}{ Group } & Coasting & $7(17 \%)$ & $23(56.1 \%)$ & $9(22.5 \%)$ & $2(4.9 \%)$ & $1.7 \pm 0.8$ \\
\hline & & Aspiration & $2(4.9 \%)$ & $14(34.2 \%)$ & $19(46.3 \%)$ & $6(14.6 \%)$ & $1.1 \pm 0.7$ \\
\hline & \multicolumn{2}{|c|}{$P$ value } & \multicolumn{4}{|c|}{0.015} & 0.0015 \\
\hline \multirow{4}{*}{ Vomiting } & \multicolumn{2}{|c|}{ Score items } & 0 & 1 & 2 & 3 & Total \\
\hline & \multirow{2}{*}{ Group } & Coasting & $14(34.2 \%)$ & $16(39 \%)$ & $11(26.8 \%)$ & 0 & $1 \pm 0.8$ \\
\hline & & Aspiration & $21(51.2 \%)$ & $17(41.5)$ & $3(7.3 \%)$ & 0 & $0.6 \pm 0.6$ \\
\hline & \multicolumn{2}{|c|}{$P$ value } & \multicolumn{4}{|c|}{0.049} & 0.009 \\
\hline \multirow{4}{*}{ Pain } & \multirow[b]{3}{*}{ Group } & items & 2 & 3 & 4 & 5 & Total \\
\hline & & Coasting & $10(24.4 \%)$ & $17(41.5)$ & $11(26.8 \%)$ & $3(7.3 \%)$ & $3.2 \pm 0.9$ \\
\hline & & Aspiration & $19(46.3 \%)$ & $16(39 \%)$ & $5(12.2 \%)$ & $1(2.5 \%)$ & $2.7 \pm 0.8$ \\
\hline & \multicolumn{2}{|c|}{$P$ value } & \multicolumn{4}{|c|}{0.108} & 0.007 \\
\hline
\end{tabular}

Data are presented as numbers \& percentages; P indicates insignificance of difference between both groups. 


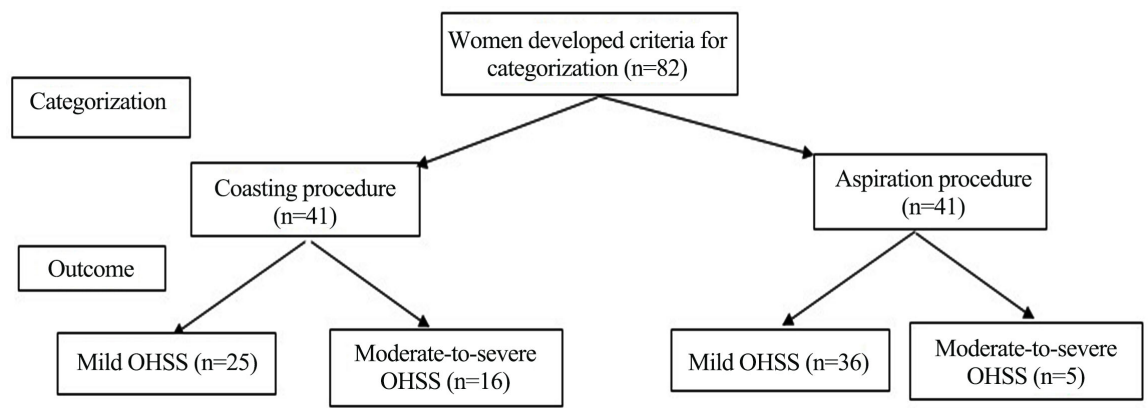

Figure 1. Consort Flow sheet.

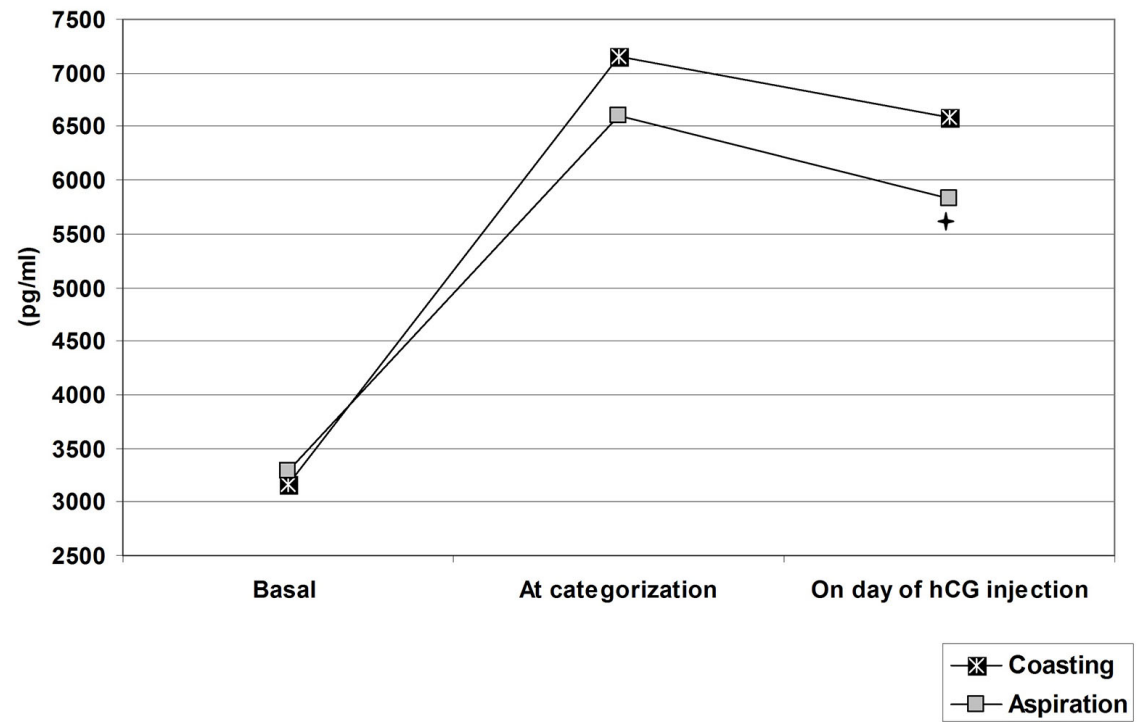

Figure 2. Mean serum E2 levels estimated in patients of both groups ( + indicates significant inter-group difference).

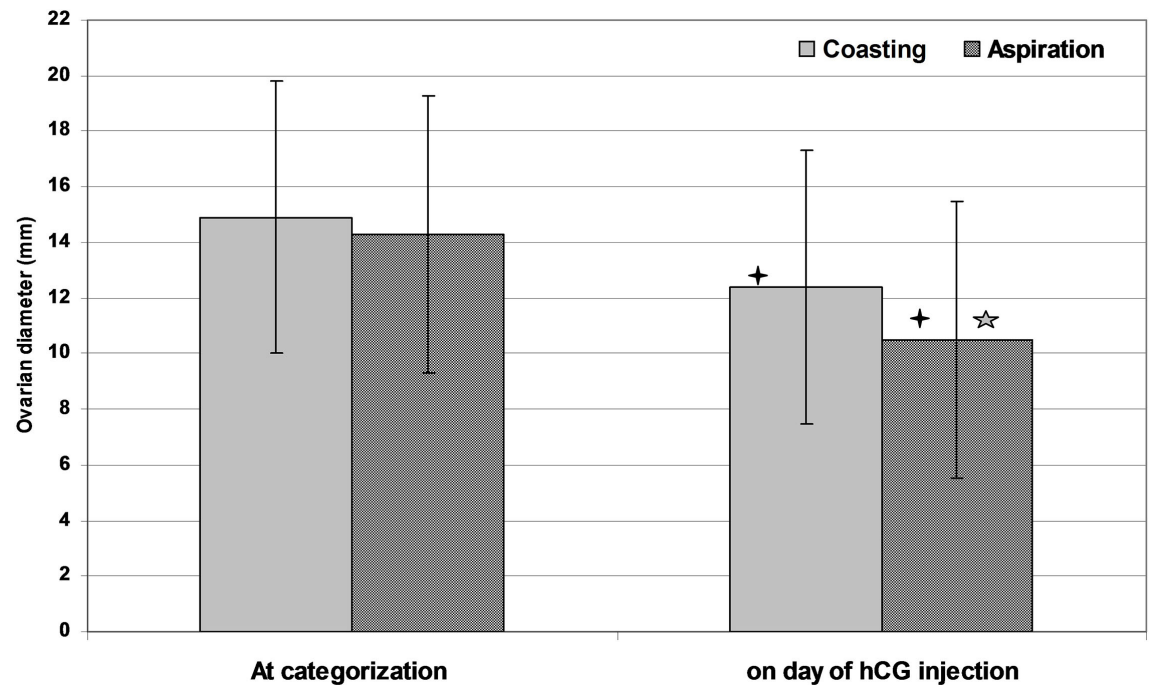

Figure 3. Mean $( \pm S D)$ ovarian diameter estimated at time of cate gorization and on day of hcG injection in women of both groups $(+$ significant difference versus at categorization; $i$ significant inter-group difference). 


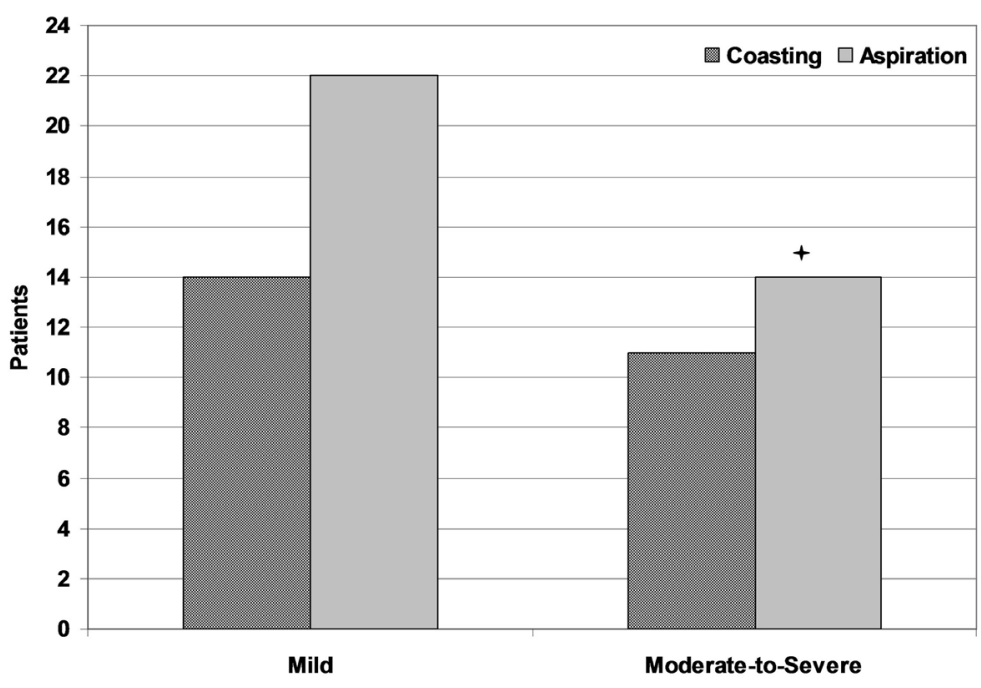

Figure 4. Patients' distribution according to severity of OHSS ( + indicates significant inter-group difference).

\section{Discussion}

The therapeutic policies provided for patients of both groups did favorably as manifested by the significant reduction of serum E2 and ovarian diameter on day of hCG injection in comparison to estimates taken at time of categorization. These results point to a fact that in women at high-risk for development of OHSS, certain therapeutic modalities could ameliorate or lessen its manifestations down to acceptable level. In support of this assumption, no patient developed severe OHSS of grade 5, only three women developed clinically detectable ascites, but no patient required paracentesis. Furthermore, about $75 \%$ of patients developed mild symptoms ranging between grade one and two.

The obtained results concerning patients received coasting procedure are in line D'Angelo et al. [23] who reviewed RCT assessed the effect of coasting on prevention of OHSS and reported lower rates of OHSS with coasting versus no coasting by rates suggesting that if $45 \%$ of women developed moderate or severe OHSS without coasting, only $4 \%-17 \%$ would develop it with coasting. Also, Abide Yayla et al. [24] in their retrospective study of women used coasting to avoid OHSS in 100 high-risk women and documented that all women had reached to embryo transfer stage and reported clinical pregnancy rate of $44 \%$. Also, Kailasam et al. [25] prospectively, found coasting for 6 days with subsequent blastocyst transfer may be an effective strategy for patients at risk of OHSS with no detrimental effects on blastocyst development or live birth outcome

Concerning comparative study for coasting and follicular aspiration as preventive strategy for OHSS, D'Angelo et al. [25] reviewed literature for RCT assessing the efficacy of coasting versus unilateral follicular aspiration and documented that data were not pooled due to heterogeneity. Moreover, review of recently published articles detected only one study compared the effect of coasting versus aspiration on incidence of OHSS; however, that study reported no difference between follicular reduction prior to HCG and coasting, in terms of OHSS 
reduction as a primary endpoint [26], these results are contradictory to the result of the current study and this difference could be attributed to multiple imitations of Bushaqer's study [26], firstly, the sample size is small; 39 women were divided into two study groups and 8 women developed OHSS; 6 versus 2 women in each group, respectively but these numbers could not reach the appropriate limit for statistical analysis to be significant or non-significant. Secondly, Bushaqer's study [26] did not comment on the frequencies of OHSS severity manifestations, which are more important than the sole frequency of OHSS.

On the other hand, the current study reported significantly reduced OHSS manifestations, both as frequency and severity, with follicular aspiration than with coasting, so the current study approved the efficacy and safety of follicular aspiration in comparison to coasting despite of proved efficacy of coasting. In support of these findings, an early study conducted by Zhu et al. [27] had documented that follicular aspiration during the selection phase can prevent severe OHSS and reduce OHSS prevalence in patients with PCOS undergoing $\mathrm{COH}$. More recently, Haydardedeoglu et al. [28] documented that in poor responders both follicular aspiration and flushing has similar results, but follicular flushing is time consuming.

The reported clinical effectiveness of coasting could be attributed to affecting follicles, especially immature follicles, through apoptosis with significant decrease in VEGF expression and secretion [29] [30]. Thus, coasting acts through inhibition of VEGF protein transcription and translation which is a time-consuming process and is unpredictable to be absolute inhibition. On the other hand, follicular aspiration allows removal of the already formed VEGF thus rapidly eliminating its effect and giving no chance by oocyte removal for re-synthesis of growth factors [31] [32]. Additionally, aspiration removes other cytokines that may have a role in induction of OHSS as IL-2 which increased levels induce vascular dilatation syndrome [33]. IL-17 [34] melatonin and melatonin receptor 2 [3] that were reported to be correlated with incidence and severity of OHSS. These data could explain the reported superior clinical effect of aspiration in comparison to coasting procedure.

\section{Conclusion}

Coasting procedure prior to hCG injection could decrease the incidence of OHSS and lessens its manifestation. However, follicular aspiration provided more superior results and improved outcome of these women. Any of these modalities could be provided to infertile high-risk women according to the availability of experiences and patients' selection. Wider scale studies are mandatory to establish the results and to evaluate underlying mechanisms for the reported clinical effectiveness of these procedures.

\section{Conflicts of Interest}

The authors declare no conflicts of interest regarding the publication of this paper. 


\section{References}

[1] Fischer, R., Nakano, F.Y., Roque, M., Bento, F.C., Baukloh, V. and Esteves, S.C. (2019) A Quality Management Approach to Controlled Ovarian Stimulation in Assisted Reproductive Technology: The "Fischer Protocol". Panminerva Medica, 61, 11-23. https://doi.org/10.23736/S0031-0808.18.03549-8

[2] Atilgan, R., Pala, Ş., Yavuzkır, Ş., Başpınar, M., Yılmaz, M. and Ilhan, N. (2019) What Is the Impact of Short- and Long-Term Supplementation of Either Cabergoline or Clarithromycin on Resolving Rat Ovarian Hyperstimulation Syndrome (OHSS) Model? Journal of Obstetrics and Gynaecology, 1-8. https://doi.org/10.1080/01443615.2018.1563774

[3] Li, Y., Fang, L., Yu, Y., Shi, H., Wang, S., Guo, Y. and Sun, Y. (2019) Higher Melatonin in the Follicle Fluid and MT2 Expression in the Granulosa Cells Contribute to the OHSS Occurrence. Reproductive Biology and Endocrinology, 17, 37. https://doi.org/10.1186/s12958-019-0479-6

[4] Stouffs, K., Daelemans, S., Santos-Ribeiro, S., Seneca, S., Gheldof, A., Gürbüz, A.S., De Vos, M., Tournaye, H. and Blockeel, C. (2019) Rare Genetic Variants Potentially Involved in Ovarian Hyperstimulation Syndrome. Journal of Assisted Reproduction and Genetics, 36, 491-497. https://doi.org/10.1007/s10815-018-1372-5

[5] Nenonen, H.A., Lindgren, I.A., Prahl, A.S., Trzybulska, D., Kharraziha, I., Hultén, M., Giwercman, Y.L. and Henic, E. (2019) The N680S Variant in the Follicle-Stimulating Hormone Receptor Gene Identifies Hyperresponders to Controlled Ovarian Stimulation. Pharmacogenetics and Genomics. https://doi.org/10.1097/FPC.0000000000000374

[6] Awonuga, A.O., Wheeler, K., Thakur, M., Jeelani, R., Diamond, M.P. and Puscheck, E.E. (2018) The Value of Delaying hCG Administration to Enable Maturation of Medium-Sized Follicles in Patients Undergoing Superovulation for IVF/ICSI. Journal of Assisted Reproduction and Genetics, 35, 289-295. https://doi.org/10.1007/s10815-017-1056-6

[7] Li, Z., Wang, A.Y., Bowman, M., Hammarberg, K., Farquhar, C., Johnson, L., Safi, N. and Sullivan, E.A. (2019) Cumulative Live Birth Rates Following a "Freeze-all" Strategy: A Population-Based Study. Human Reproduction Open, 2019, hoz004. https://doi.org/10.1093/hropen/hoz004

[8] Kan, O., Simsir, C., Atabekoglu, C.S. and Sonmezer, M. (2019) The Impact of Adding hp-hMG in r-FSH Started GnRH Antagonist Cycles on ART Outcome. Gynecological Endocrinology. https://doi.org/10.1080/09513590.2019.1600667

[9] Fernández-Sánchez, M., Visnova, H., Yuzpe, A., Klein, B.M., Mannaerts, B. and Arce, J.C. (2019) ESTHER-1 and ESTHER-2 Study Group: Individualization of the Starting Dose of Follitropin Delta Reduces the Overall OHSS Risk and/or the Need for Additional Preventive Interventions: Cumulative Data over Three Stimulation Cycles. Reproductive BioMedicine Online, 38, 528-537. https://doi.org/10.1016/j.rbmo.2018.12.032

[10] Guo, J.L., Zhang, D.D., Zhao, Y., Zhang, D., Zhang, X.M., Zhou, C.Q. and Yao, S.Z. (2016) Pharmacologic Interventions in Preventing Ovarian Hyperstimulation Syndrome: A Systematic Review and Network Meta-Analysis. Scientific Reports, 6, Article ID: 19093. https://doi.org/10.1038/srep19093

[11] Turan, G.A., Eskicioglu, F., Sivrikoz, O.N., Cengiz, H., Adakan, S., Gur, E.B., Tatar, S., Sahin, N. and Yilmaz, O. (2015) Myo-Inositol Is a Promising Treatment for the Prevention of Ovarian Hyperstimulation Syndrome (OHSS): An Animal Study. Archives of Gynecology and Obstetrics, 292, 1163-1171. 
https://doi.org/10.1007/s00404-015-3747-5

[12] Turan, G.A., Eskicioglu, F., Sivrikoz, O.N., Cengiz, H., Gur, E.B., Tatar, S., Sahin, N. and Yilmaz, O. (2015) Prophylactic Vitamin D Supplementation in Ovarian Hyperstimulation Syndrome: An Animal Study. Archives of Gynecology and Obstetrics, 292, 421-427. https://doi.org/10.1007/s00404-015-3625-1

[13] Akman, L., Sahin, G., Erbas, O., Aktug, H., Akdogan, A., Goker, E.N., Taskiran, D. and Tavmergen, E. (2015) Comparison of Montelukast and Cabergoline for Prevention of Ovarian Hyperstimulation Syndrome: In an Experimental Rat Model.

[14] Kasap, E., Turan, G.A., Eskicioğlu, F., Cengiz, H., Gur, E.B., Sivrikoz, O.N., Genc, M. and Yilmaz, O. (2016) Comparison between Resveratrol and Cabergoline in Preventing Ovarian Hyperstimulation Syndrome in a Rat Model. Gynecological Endocrinology, 32, 634-640. https://doi.org/10.3109/09513590.2016.1152575

[15] Kailasam, C., Griffith, H., Wilson, P. and Gordon, U. (2018) The Effect of Early Coasting on Blastocyst Development and Outcome Following Blastocyst Transfer in IVF/ICSI Programme. JBRA Assisted Reproduction, 22, 301-306. https://doi.org/10.5935/1518-0557.20180053

[16] Galal, A.F., Abbassy, H.A., AbdElrahman, A.H., Abdelhafez, M.S. and Badawy, A. (2018) Accelerated Coasting Does Not Affect Oocyte Maturation. Gynecologic and Obstetric Investigation, 83, 145-150. https://doi.org/10.1159/000477297

[17] Rotterdam ESHRE/ASRM-Sponsored PCOS Consensus Workshop Group (2004) Revised 2003 Consensus on Diagnostic Criteria and Long-Term Health Risks Related to Polycystic Ovary Syndrome. Fertility and Sterility, 81, 19-25. https://doi.org/10.1016/j.fertnstert.2003.10.004

[18] Huisman, G.J., Fauser, B.C., Eijkemans, M.J. and Pieters, M.H. (2000) Implantation Rates after in Vitro Fertilization and Transfer of a Maximum of Two Embryos That Have Undergone Three to Five Days of Culture. Fertility and Sterility, 73, 117-122. https://doi.org/10.1016/S0015-0282(99)00458-6

[19] Steer, C.V., Mills, C.L., Tan, S.L., Campbell, S. and Edwards, R.G. (1992) The Cumulative Embryo Score: A Predictive Embryo Scoring Technique to Select the Optimal Number of Embryos to Transfer in an In-Vitro Fertilization and Embryo Transfer Programme. Human Reproduction, 117, 7-9. https://doi.org/10.1093/oxfordjournals.humrep.a137542

[20] Golan, A., Ron-El, R. and Herman, A. (1989) Ovarian Hyperstimulation Syndrome: An Update Review. Obstetrics \& Gynecology Science, 44, 430-440. https://doi.org/10.1097/00006254-198906000-00004

[21] Aboulghar, M.A. and Mansour, R.T. (2003) Ovarian Hyperstimulation Syndrome: Classifications and Critical Analysis of Preventive Measures. Human Reproduction Update, 9, 275-289. https://doi.org/10.1093/humupd/dmg018

[22] Scott, J. Huskisson, E.C. (1976) Graphic Representation of Pain. Pain, 2, 175-184. https://doi.org/10.1016/0304-3959(76)90113-5

[23] D’Angelo, A., Amso, N.N. and Hassan, R. (2017) Coasting (Withholding Gonadotrophins) for Preventing Ovarian Hyperstimulation Syndrome. Cochrane Database of Systematic Reviews, No. 5, CD002811. https://doi.org/10.1002/14651858.CD002811.pub4

[24] Abide Yayla, C., Ozkaya, E., Beydilli Nacak, G., Sanverdi, I., Devranoglu, B., Bostanci Ergen, E., Kilicci, C. and Kutlu, T. (2018) High Estrogen Exposure May Not Be Detrimental on Endometrial Receptivity in Women with PCOS. Gynecological Endocrinology, 34, 798-803. https://doi.org/10.1080/09513590.2018.1460344 
[25] D’Angelo, A., Brown, J. and Amso, N.N. (2011) Coasting (Withholding Gonadotrophins) for Preventing Ovarian Hyperstimulation Syndrome. Cochrane Database of Systematic Reviews, No. 6, CD002811. https://doi.org/10.1002/14651858.CD002811.pub3

[26] Bushaqer, N.J., Dayoub, N.M., AlHattali, K.K., Ayyoub, H.A., AlFaraj, S.S. and Hassan, S.N. (2018) Follicular Aspiration versus Coasting for Ovarian Hyper-Stimulation Syndrome Prevention. Saudi Medical Journal, 39, 290-295. https://doi.org/10.15537/smj.2018.3.22331

[27] Zhu, W.J., Li, X.M., Chen, X.M. and Zhang, L. (2005) Follicular Aspiration during the Selection Phase Prevents Severe Ovarian Hyperstimulation in Patients with Polycystic Ovary Syndrome Who Are Undergoing in Vitro Fertilization. European Journal of Obstetrics \& Gynecology and Reproductive Biology, 122, 79-84. https://doi.org/10.1016/j.ejogrb.2005.01.023

[28] Haydardedeoglu, B., Gjemalaj, F., Aytac, P.C. and Kilicdag, E.B. (2017) Direct Aspiration versus Follicular Flushing in Poor Responders Undergoing Intracytoplasmic Sperm Injection: A Randomised Controlled Trial. BJOG, 124, 1190-1196. https://doi.org/10.1111/1471-0528.14629

[29] García-Velasco, J.A., Zúñiga, A., Pacheco, A., Gómez, R., Simón, C., Remohí, J. and Pellicer, A. (2004) Coasting Acts through Downregulation of VEGF Gene Expression and Protein Secretion. Human Reproduction, 19, 1530-1538. https://doi.org/10.1093/humrep/deh298

[30] Abdallah, R., Kligman, I., Davis, O. and Rosenwaks, Z. (2010) Withholding Gonadotropins until Human Chorionic Gonadotropin Administration. Seminars in Reproductive Medicine, 28, 486-492. https://doi.org/10.1055/s-0030-1265675

[31] Gao, M.Z., Zhao, X.M., Sun, Z.G., Hong, Y., Zhao, L.W., Zhang, H.Q. (2011) Endocrine Gland-Derived Vascular Endothelial Growth Factor Concentrations in Follicular Fluid and Serum May Predict Ovarian Hyperstimulation Syndrome in Women Undergoing Controlled Ovarian Hyperstimulation. Fertility and Sterility, 95, 673-678. https://doi.org/10.1016/j.fertnstert.2010.09.044

[32] Manau, D., Fábregues, F., Peñarrubia, J., Creus, M., Carmona, F., Casals, G., Jiménez, W. and Balasch, J. (2007) Vascular Endothelial Growth Factor Levels in Serum and Plasma from Patients Undergoing Controlled Ovarian Hyperstimulation for IVF. Human Reproduction, 22, 669-675. https://doi.org/10.1093/humrep/del427

[33] Orvieto, R., Dratviman-Storobinsky, O. and Cohen, Y. (2015) Interleukin-2 Production by Cultured Human Granulosa Cells. American Journal of Reproductive Immunology, 74, 392-397. https://doi.org/10.1111/aji.12416

[34] Motamedzadeh, L., Mohammadi, M.M., Hadinedoushan, H., Farashahi Yazd, E. and Fesahat, F. (2019) Association of IL-17 and IL-23 Follicular Fluid Concentrations and Gene Expression Profile in Cumulus Cells from Infertile Women at Risk for Ovarian Hyperstimulation Syndrome. Human Fertility (Cambridge, England), 1-7. https://doi.org/10.1080/14647273.2019.1566648 